\title{
POLÍTICA SOCIAL PARA CIUDADANOS CON CAPACIDADES FÍSICAS Y MENTALES REDUCIDAS Y EN SITUACIÓN DE CALLE
}

\author{
Rosa Soto Yánez, ${ }^{1}$ \\ Liliana Pérez Mendoza ${ }^{2}$
}

\section{Resumen}

El artículo está basado en una propuesta para una política pública dirigida a ciudadanos con capacidades físicas y mentales reducidas y que se encuentren en situación de calle, para el caso del contexto chileno. Incluye aportes como el planteamiento del problema, el enfoque, los objetivos, los principios, las estrategias, las opciones, los criterios de evaluación, el diseño e implementación de la misma.

Descriptores: Capacidades físicas y mentales reducidas, ciudadanos en situación de calle, política social.

\begin{abstract}
The article is based on a proposal for a public policy aimed for homeless people with low physical and mental capacities, in the case of the Chilean context. Include sections such as the approach to the problem, focus, goals, principles, strategies, options, evaluation criteria, it's design and implementation.
\end{abstract}

Key words: Reduced physical and mental abilities, homeless people, social policy.

\section{Contexto y planteamiento del problema}

En las últimas décadas en América Latina y específicamente, en Chile se han suscitado grandès transformaciones básicamente en dos ejes centrales: los procesos de democratización y el fenómeno de la modernización, los cuales han implicado la tendencia a pasar a una economía global y segmentada. No es nuevo que los procesos económicos sean mundiales, pero si lo es, la presencia de una economía en que cotidianamente sus unidades de funcionamiento y de gestión están interrelacionadas

\footnotetext{
${ }^{1}$ Trabajadora social sistema Judicial de Rancagua, Chile.

${ }^{2}$ Docente-investigadora de la Facultad de Ciencias Sociales y Educación de la Universidad de Cartagena, Colombia.
} 
Este orden económico mundial, productivo en gran medida, segmenta, dejando de lado a grandes proporciones de población mundial, surgiendo lo que se conoce como las nuevas exclusiones sociales, que provocan la expulsión de grandes porcentajes de población económicamente activa, nuevas formas en las relaciones de producción, una escasa o nula vinculación entre el crecimiento de la producción y el empleo y una mayor desigualdad entre ricos y pobres. Es decir, Un Estado de Bienestar en crisis, cuyas contradicciones están entre el desarrollo capitalista y los derechos construidos en el proyecto de la sociedad moderna, que van enmarcando lo que se conoce como la nueva cuestión social ${ }^{3}$.

La globalización convertida en ideología y pensamiento único, se ha vuelto globalismo, o sea imposición de la globalización de los mercados y reducción al mercado de las discrepancias políticas y las diferencias culturales. Al subordinar estos dos escenarios de la diferencia a una sola visión de la economía, lo político se diluye y el Estado parece casi innecesario (García, 2001: 180).

Es así como, del estado regulador, integrador de políticas sociales de carácter más universal, se pasa a un Estado expulsor de importantes sectores de la población y animador de políticas sociales tendientes a recortar el gasto público, básicamente aquel que incluye a los usuarios de los sectores más desfavorecidos de la sociedad, como salud, educación y previsión social.

Conformándose así nuevas dimensiones que forma a este escenario son: la configuración de las nuevas redes sociales; las nuevas relaciones entre el Estado y la sociedad civil; el surgimiento de otros actores políticos; la crisis de representatitividad; las nuevas formas organizativas y de poder económico-político, mercados regionales y movimientos sociales de nuevo tipo.

Esto entre otras cosas, provoca, y a su vez es provocado por una crisis del sistema institucional cuyas características centrales pasan a ser la agudización de la corrupción y la distancia entre la palabra y la acción. Las instituciones incluidas la familia, la sociedades de vecinos, los sindicatos y demás organizaciones se debilitan en su función integradora y hoy es posible visualizar en ellas elementos desintegradores, que dan lugar a nuevas problemáticas sociales sobre todo en las grandes ciudades, y a la ruptura del vinculo social que caracterizó a las sociedades organizadas en el marco del estado regulador.

\footnotetext{
${ }^{3}$ Según Carlos Eroles, "Hablar de la cuestión social es centrar desde el contexto socio-económico, político y cultural la realidad que viven nuestros pueblos. Esta realidad está signada por los mayores índices de desigualdad del planeta. Nuestro continente afronta significativos índices de pobreza, que se ubican para muchos países en un porcentaje superior al $40 \%$ de la población total. Pero estos índices, salvo excepciones, se van modificando hacia abajo, a medida que se produce una mayor incidencia de los procesos de desarrollo. Sin embargo lo que no disminuyen son los niveles de indigencia y desigualdad social, que conducen inevitablemente al conocido fenómeno de la exclusión social" (2006: 1)
} 
Pero así como el fenómeno de la globalización ha cruzado los ejes económicos, políticos, sociales, culturales, es posible desde las ciencias sociales reconocer y rescatar dentro de los sectores más marginados esbozos aún presentes de solidaridad, entendida como la creación de oportunidades y desarrollo de las capacidades para todos, que intentan de algún modo, producir inclusiones o inserciones de personas que la única forma de seguir haciendo parte de una ciudadanía es la conformación o fortalecimiento de una red de tipo social, que pueda contribuir a su autonomía y ejercicio pleno sus derechos ciudadanos.

En el caso chileno, hoy en día, se muestra la coexistencia de grupos humanos que se han visto tremendamente beneficiados con los procesos generados por la globalización, pero también esto mismo ha posibilitado la formación de grupos humanos que se clasifican como 'los sin techo', 'los delincuentes', 'los alcohólicos'. 'los desechables', 'locos', 'humanidad excedente' ó lo que Iamamoto (2002) llama "los descartables" (p.34-35). Esta semántica, explica el surgimiento de categorizaciones y estereotipos creados en general, a partir de atributos negativos. Ellos no sólo son asumidos como diferentes, lo que por si sólo ya implica una toma de distancia, sino que además se entiende como que pueden llegar a ser peligrosos, constituyéndose desde un punto de vista policivo ó represivo, una virtual amenaza para la comunidad. Ahora, desde el gobierno central y desde el local, esta situación está invisibilizada por lo tanto no es abordada como un fenómeno existente y con características crecientes en la sociedad. $\mathrm{Y}$ es que estas personas no son consideradas siquiera como excluidas sino como "sobrantes", en este sentido, se retoma lo señalado por Carlos Eroles (2006):

Solo el excluido visibiliza derechos, el sobrante no, ya que no registra que tenga algo que reclamar, justamente por estar fuera de las relaciones constructoras de 'ciudadanía'. Por lo tanto en este caso no nos encontramos con vulneración de derechos, ya que el derecho no existe, en tanto construcción subjetiva (p.2).

Es decir, que no se representan socialmente como ciudadanos con derecho a reclamar derechos.

Un ejemplo patético de esta situación es que en Chile no existe un censo de la gente adulta con capacidades físicas y mentales reducidas y que se encuentra en situación de calle, que de cuenta de que al menos hay una preocupación por reconocer este problema, desconociéndose el número y la situación en que viven las personas en la calle.

En el mes de Octubre del año 2003, la Fundación Hogar de Cristo en conjunto con el MIDEPLAN (2003) realizó en la Comuna de Estación Central un Censo de las Personas que vivía en situación de calle, registrándose alrededor de 526 personas entre niños, mujeres y adultos, solamente en ese lugar. Del total de esas 526 personas, el $68.1 \%$ correspondió a hombres y el $31.9 \%$ a mujeres. De este total el $42.5 \%$ eran 
hombres entre 30 a 59 años y el $50.6 \%$ correspondió a mujeres en el mismo rango etáreo. El 68.1\% (358) correspondió a los hombres y el 31.9\% (168) eran mujeres, de los cuales el $89.5 \%$ (418) decían saber leer y escribir, mientras que un $10.1 \%$ que no sabían leer ni escribir, lo cual permite asegurar que este aprendizaje resulta básico para el desarrollo de acciones educativas que pretendan la habilitación social de esta población. La mayoría (84.9\%) se encontraba sin pareja, lo cual sin duda es un agravante para el desarrollo de acciones que involucren el acompañamiento familiar. Es relevante destacar que la mayoría (46.8\%) asumían que llevaban un año o menos de vivir en la calle, en tanto, que el $22.7 \%$ sostenía que llevaba entre dos a cinco años de estar en la calle. Esto permite hipotetizar que el problema se ha agravado en los últimos años, dado el porcentaje que alcanzó con relación a los años anteriores que suman un promedio de $45.6 \%$ que tenía entre dos a dieciséis años y un $7.5 \%$ que decía no recordar desde cuando estaba la calle.

El Censo de MIDEPLAN (2003) también refleja que el 74.6\% de esta población duerme en Hospederías solidarias, mientras que el $11.2 \%$ decía que en una residencia solidaria, el $7.3 \%$ en residencias temporales y un $6.7 \%$ dormían en la vía pública. El $26.8 \%$ afirmó que tuvieron contacto con su familia en menos de una semana, mientras que un $20.9 \%$ sostuvo que su contacto familiar fue entre un mes o menos de un año, siendo el porcentaje más alto $28.9 \%$ (152 personas) que afirmaron que hace más de un año que tuvieron el último contacto con su familia. Es decir, el contacto con la familia más frecuente va de una semana hasta un año, lo cual induce a pensar que durante el primer año la familia permanecía atenta a su familiar en situación de calle, pero después de éste tiempo el contacto se iba disminuyendo.

Con relación a servicios, el $82.7 \%$ (435) sostuvo que tenía acceso a éstos, cifra alta, pues eso podría indicar que el mayor porcentaje de esta población estaría cubierta por algún tipo de servicio. Sin embargo, estas personas continúan en situación de calle desde hace algún tiempo. De esos servicios el $57.8 \%$ afirmó que este era prestado por una organización de beneficencia, mientras que el $18.6 \%$ señaló que lo prestaban las iglesias o templos y sólo el $12.4 \%$ lo ofrecía la Municipalidad, mientras que el resto lo brindaban los hospitales, consultorios, establecimientos educacionales y otros (MIDEPLAN, 2003). Esta situación permite deducir que son los organismos de beneficencia social y religiosa, quienes en mayor medida asumen la asistencia a esta población, por ello el problema es ignorado desde el Estado y la atención que se ofrece es de caridad, más no de servicios en su forma natural de prestación.

Del total de la población entre 30 y 59 años, el $52.3 \%$ afirmó que antes de vivir en esta situación vivía en su casa, lo cual se constituyó en más de la mitad de los casos, con ello podríamos decir que es la familia la que se estaría encargando de expulsar a sus miembros a la calle, ante cualquier disminución de las capacidades físicas o mentales de alguno de ellos por la incapacidad de atenderlos. El siguiente porcentaje, es decir el $5.2 \%$ afirmó que vivía en casa de otro familiar, un amigo o un conocido lo cual 
reafirma lo anterior y además, da lugar a pensar que la casa de otros familiares y amigos pasa a ser considerada el segundo espacio en que se ubican éstas personas, antes de llegar a la calle. Por lo tanto, la red familiar es un paso intermedio entre la salida del hogar y la llegada a la calle. Sólo el $15.7 \%$ afirmó que antes estaba en un lugar internado (cifra que tiende a aumentar por el tema de la reforma siquiátrica), en una pieza o en una casa arrendada.

Es importante mencionar que el mayor porcentaje (30.7\%) de la población en situación de calle afirmó estar allí por su situación económica (MIDEPLAN, 2003), es decir que tal vez la falta de acceso a un lugar de trabajo o el nulo aporte a la economía familiar incide para que sea expulsado por ésta, situación a considerar, pues es necesaria entonces una política que utilice también su capacidad en la búsqueda de algún ingreso económico, a fin de éste pueda reintegrarse a la familia. El 24.6\% afirmó que estaba en la calle por problemas con su familia, y en esta medida se tiene entonces, que la red familiar no estaría siendo solidaria con estos miembros. Por lo tanto, éste debería ser también un factor a trabajar. Un $15.6 \%$ mencionó el consumo de droga o problemas mentales (MIDEPLAN, 2003), en este caso es un porcentaje que habría de trabajar con mayor énfasis, porque se está frente a un problema que genera mayor resistencia de la familia y la comunidad para su aceptación y habilitación social. El 9.3\% afirmó que estaba en la calle por problemas de salud física, mientras que el $4.2 \%$ no tenía una casa, un hogar, una familia donde llegar, en tal caso, se estaría frente a personas que adolecen de toda red familiar o comunitaria, lo cual implica un tipo de respuestas que los incorpore a espacios donde vivir (MIDEPLAN, 2003). Este número tiende a crecer, pues la Reforma Psiquiátrica ha iniciado un proceso de desintitucionalización de pacientes con larga estadía y ninguna institución en particular asume la atención de personas enfermas, pues el Servicio de Salud los egresa rápidamente, no existiendo lugar para acogerlos y atender sus necesidades.

El discurso dominante en el país con relación al tema ha sido el siguiente:

- El tema de la salud en Chile pasa a ser de responsabilidad de las familias y comunidades, sale del área de la salud, por lo tanto hay una progresiva mirada hacia el tema psiquiátrico por fuera de la política estatal, y por ende de atención en instituciones del Estado.

- La situación de indigencia y su desvinculación con la red social y familiar impide el acceso a los programas y organizaciones gubernamentales.

- El rango de hombre y mujeres entre 30 y 49 años no son considerados como grupos priotarios para la política social (los que se consideran son los grupos poblacionales como: Niños, jóvenes, mujeres jefas de hogar y adultos mayores).

- El enfermo mental permanece excluido socialmente debido a que la población no ha sido educada en la comprensión de estos trastornos, sino en la concepción de que es un trastorno crónico con carga genética y tratado farmacológicamente. 
De otro lado, se tienen las siguientes percepciones respecto de las personas con capacidades físicas y mentales reducidas:

- Estas personas se asumen categóricamente como incapaces de incorporarse a la actividad productiva del país.

- No existe una consideración respecto a que tienen derechos y con capacidades instaladas para ser útiles en la sociedad.

- La atención se mira desde el punto de vista del fármaco y desde las debilidades mentales y no desde sus potencialidades y la necesidad de habilitación social desde lo ocupacional.

- Persiste en buena parte una discriminación de la atención a la salud mental en el Sistema General de Salud.

Las políticas existentes orientadas a adultos con capacidades físicas y mentales reducidas en el país han sido las siguientes:

- El plan auge contempla en la modalidad institucional, las prestaciones asociadas al diagnóstico y tratamiento de la esquizofrenia en el paciente de Fonasa y tiene cobertura financiera, en función de su grupo de ingreso.

- En el caso que haya una sospecha clínica del primer episodio el paciente asistirá a la primera entrevista siquiátrica ambulatoria en 20 días.

- Existencia de una reforma siquiátrica con tendencia a la desinstitucionalización de los pacientes con larga estadía en hospitales psiquiátricos.

- Existe la posibilidad de postular estas personas a pensiones asistenciales por invalidez, sin embargo en la práctica esta posibilidad es muy remota debido a los pocos cupos y a las largas listas de espera que manejan los municipios.

- Las instituciones que trabajan con adultos mayores están imposibilitadas de acoger a esta población específica por cuanto no cuentan con los recursos profesionales especializados para la atención no acogiéndolos y no ofertando alternativas de atención.

Así pues, éste es un problema público por cuanto va adquiriendo importancia en el país desde las instituciones de beneficencia que en su actuar diario se encuentran con este tipo de personas que deben atender, pero con grandes limitaciones en términos de recursos humanos especializados e insumos materiales y locativos. La limitación física y mental ha sido abordada desde el Estado creando incluso el Fondo Nacional de la Discapacidad, sin embargo coexiste este segmento de personas que se encuentran en situación de calle y que no acceden a estos beneficios de la red estatal.

El problema alcanza magnitudes relevantes por cuanto las primeras aproximaciones del estudio realizado en la Comuna de Estación Central (MIDEPLAN,2003), reveló que existían 526 personas entre niños, mujeres y adultos en situación de calle, destacándose que el $9.3 \%$ afirmaba que estaba en la calle por problemas de salud 
física, el $15.5 \%$ dicía que su situación se debía a problemas de consumo de alcohol, drogas o problemas mentales. De esta población el $46.8 \%$ asumía que llevaba un año de vivir en la calle, en tanto que el $22.7 \%$ tenía entre dos a cinco años de estar en la calle. A diario en el país, a través de los medios de comunicación esta situación adquiere notoriedad asociada a la participación de estas personas en hechos delictuales y además, visibiliza de manera extrema la pobreza y sus dimensiones.

La inexistencia de redes familiares y sociales de estas personas agrava más el problema por cuanto impide la representación de esta demanda social desde los mismos sujetos afectados, haciendo que no pase de ser visto como una situación que afecta más a individuos aislados que a grupos o colectivos de personas, que reflejan de algún modo el deterioro de la calidad de vida de algunos grupos poblacionales. Para analizar el problema se tienen en cuenta varios criterios de acuerdo a la perspectiva de los diferentes actores partícipes de la situación es así como, desde la perspectiva de los sujetos, se señalan los criterios de agregación, organización, representación y de concertación.

En cuanto al primero, se tiene que para las Fundaciones que trabajan con personas vinculadas al mundo de la extrema pobreza el problema es importante, pues se constituyen en sí en la única alternativa de atención para éstas personas, pues no existen redes que acojan de manera integral el problema que le aqueja. Por otra parte, este segmento de población se constituye en un desafío y permanente cuestionamiento, pues en su interior no disponen de los recursos necesarios para una atención integral, exigiendo el destino de mayores recursos para su adecuada atención con los cuales no cuentan.

Con relación al criterio de organización y representación, este segmento de población dada su misma condición de capacidades físicas y mentales reducidas se convierte en un factor que contribuye a una mayor exclusión social por lo tanto con menos capacidad de explicitar sus demandas a través de organizaciones y redes de apoyo familiar y social. Lo que quizás pudiera ayudar a asumir este problema en términos de su visibilización en el contexto social es a través de un trabajo de reconfiguración de sus redes sociales y de una mayor presión de las entidades que asumen de manera voluntaria y benéfica su atención, pero éstas no han respondido sostenidamente con estrategias que puedan lograr efectos como la reinserción social de esta personas, pues la atención es en términos de asistencia únicamente.

Finalmente, se encuentra el criterio de concertación para analizar desde los sujetos el problema: desde la perspectiva de las instituciones benéficas y voluntariados, se asume su atención por condiciones específicamente de solidaridad social, pero esta atención no trasciende a un nivel público pues no existen acciones concertadas de manera interinstitucional, que contribuyan al menos, a difundir esta necesidad y mucho menos crear alianzas estratégicas entre ellas y con el Estado que contribuyan a afrontar esta realidad, diseñando estrategias de intervención que no sólo atenúen el problema sino que lo prevengan. 
Desde el escenario del problema, se tienen en cuenta criterios como los de Legitimación, Aceptación, Conflictividad y Permisibilidad. En cuanto al primer criterio, el problema ha sido asumido coyunturalmente por parte de instituciones de beneficencia y voluntariados que no alcanzan a interlocutar con otros actores institucionales que puedan aunar esfuerzos para una solución más integral del mismo. Es posible concertar la articulación de redes institucionales del área de la salud, del trabajo y previsión social, cuyo objeto de acción tiene que ver con el problema de las capacidades físicas y mentales reducidas.

Otro criterio importante para analizar el problema es el de aceptación social del mismo, pues la solución al problema se enfrenta principalmente con la creencia acerca de que las personas con capacidades físicas y mentales reducidas no hacen ningún aporte económico y social al país, por tanto están por fuera del imaginario social que el carácter globalizado de la sociedad exige a cada individuo. Asimismo, desde la perspectiva más individual no se reconocen habilidades ni potencialidades a esta población por lo tanto las alternativas de solución tienden por un lado a la atención médica y por otro, a la institucionalización del sujeto, más que a su reinserción social. Desde la perspectiva cultural, estas personas tienden a ser 'escondidas', 'encerradas', 'marginadas' y 'negadas' en términos de sus derechos como ciudadanos, ya sea por parte de la familia o por la sociedad.

Por otro lado, los conflictos que se podrían generar con la política están en varias dimensiones. Por un lado, con las familias que han decidido ignorar el problema de estos miembros; por otro lado, con las instituciones de salud que no cuentan con todos los servicios para atender esta demanda de manera integral; con los gobiernos locales, que niegan posibilidad de existencia en sus comunas de personas con altos niveles de exclusión social como el caso de esta población, por ejemplo. La fuerza pública, actúa en forma represiva con relación a estas personas por lo tanto un cambio en el tratamiento de esta problemática les va a significar nuevos desafíos de actuación cívica y de seguridad. Estos factores tienen que ver con un criterio de conflictividad con relación a problema.

El problema tiene que ver con otro criterio como es el de Permisibilidad, pues el tema de los derechos humanos y específicamente los de segunda generación así como las políticas de segunda generación que hoy en día cobran vigencia, posibilitan una mirada más cercana y solidaria frente a la situación de personas que pese al desarrollo económico generalizado con la globalización, se encuentran en situaciones de desigualdad que muestran las debilidades del sistema económico y la diferenciación siempre presente entre el sistema social y el económico. Por esta razón, se estima que el tema va a sensibilizar y a lograr el apoyo de los actores como son los grupos sociales organizados, partidos políticos, sectores influyentes y opinión pública permitiendo crear presión social para que el mismo empiece a ser parte de la Agenda Pública. 


\section{Enfoque de la política}

El desafío de un Estado que posibilite la inserción social es la invención de reglas para vivir una democracia que resuelva por procesos de gestión deliberativa, las condiciones bajo las cuales las situaciones particulares se hacen meritorias (por equivalencias), para una justa acción de los servicios del Estado en su resolución. En este sentido, se entiende la democracia como señala Luhmann, como el mantenimiento de la complejidad con el trabajo decisorio conjunto, es decir, como el mantenimiento del ámbito de selección más amplio posible para decisiones siempre nuevas y diversas.

Esta política está orientada hacia a hombres y mujeres entre los 30 y 59 años de edad en condiciones de abandono en la calle y con carencias de redes sociales y familiares que presenten: reducción de sus capacidades físicas y mentales, consumo de alcohol y drogas, alto deterioro cognitivo y funcional, bajos niveles de habilidades sociales y de autocuidado y largos períodos de cesantía. Las personas sujetos de la política tienden a deambular en los espacios públicos, tales cómo: lugares céntricos de la ciudad, en las plazas, en las ferias y cerca de las obras de la institución Hogar de Cristo.

Se requiere caracterizar esta población desagregando aspectos como los siguientes, a fin no sólo cuantificar la magnitud del problema sino también, de identificar los sujetos en términos de: sexo, edad, escolaridad, capacitación en arte y oficios, tiempo de estadía en la calle, historia personal, enfermedad psiquiátrica que presenta, vínculos familiares, procedencia, estrategias de supervivencia utilizadas, grupos de pertenecía, redes informales con las cuales se encuentran vinculados, grado de dificultad de acceso a familiares y a domicilios, historias clínicas, mapas de ubicación territorial, las trayectorias personales, cuadros clínicos y por último, indagar acerca de las necesidades y demandas desde estas personas frente a su situación. También, se trata de fomentar la incorporación y participación de las familias de este grupo poblacional, a fin de generar una organización que demande a la sociedad y el Estado respuestas directas $\mathrm{y}$ eficientes frente a esta realidad.

Los actores llamados a participar en la implementación de esta política son las Instituciones Privadas sin fines de lucro tales como: Fundación de Beneficencia Hogar de Cristo, a través de las Hospederías y otras fundaciones y ONG's y el mismo Estado a través de políticas como las siguientes:

- Plan Auge, que contempla atención a pacientes esquizofrénicos, a través de Fondo Nacional de Salud (FONASA).

- La Reforma a la Salud Mental, plantea la desintitucionalización de pacientes psiquiátricos de larga estadía, planteando cómo alternativa programas ambulatorios de atención, los cuales no existen.

- Desde la Seguridad Social la posibilidad de acceder a Pensiones Asistenciales por invalidez psiquiátrica, que en términos reales no es factible dado los escasos 
cupos que administran los Municipios.

- El rol subsidiario del Estado que asegura un mínimo de atención a las personas pobres, vulnerables y sin capacidad de pago. Que en la práctica se traduce en ausencia de atención

Es necesario identificar y caracterizar las instituciones públicas y privadas que ofrecen algún tipo de servicios a personas con capacidades físicas y mentales reducidas, consumo de alcohol y drogas, situación de calle o abandono de la red social y familiar, tendientes a articular acciones y recursos dirigidos a hacer más eficiente la prestación de servicios que ofrecen o nuevas posibilidades de programas. También es válido diseñar estrategias de cabildeo profesional frente a organismos del Estado e instituciones privadas, a fin de poner en la agenda pública este tema para debate y respuestas (Ministerio de Salud, Ministerio del trabajo, Ministerio de Educación, Gobiernos Locales).

Los principios que deben guiar la política social son: 1) el derecho internacional humanitario que establece el reconocimiento y el respeto por la dignidad humana; 2)reconocimiento de las personas con este tipo de limitación cómo sujetos de derechos; 3) acceso igualitario de las personas a los servicios sociales del Estado; 4) reconocimiento de que toda persona tiene capacidades y potencialidades que pueden contribuir a la superación de sus dificultades; 5) promoción de derechos y deberes ciudadanos, vida independiente, autonomía, inclusión social, el desarrollo de habilidades y destrezas de la persona con reducidas capacidades físicas y mentales y los apoyos y soportes que se ofrezcan desde la comunidad; y propiciar la organización de las familias de personas con capacidades físicas y mentales reducidas y en situación de abandono, con el ánimo de demandar de la sociedad y el Estado respuestas frente a los problemas derivados de esta situación

El objetivo general de la política social debe ser: contribuir al ejercicio de los derechos ciudadanos de hombres y mujeres con edades entre los 30 y 59 años en situación de abandono y con carencia de redes familiares y sociales, que presentan reducción de sus capacidades físicas y mentales, y con bajos niveles de habilidades sociales, integrándolos a la vida comunitaria con el compromiso de la familia, la sociedad y el Estado. Entre los objetivos específicos están: conformar una red interinstitucional desde el Estado, la sociedad civil, el tercer sector y la familia para elaborar un plan que aborde el problema desde sus diversas dimensiones (biopsicosociales); propiciar condiciones de acceso igualitario y prioritario de esta población a la atención en salud y a la red de servicios sociales del Estado y/o comunidad; promover el fortalecimiento de redes sociales y materiales para garantizar la autonomía e integración social de este sector poblacional. 


\section{Enfoque sectorial / intersectorial}

El sector de organizaciones no gubernamentales que ha tenido contacto directo o indirecto con esta población y que de alguna manera conoce sus características, en este caso es uno de los llamados a liderar esta propuesta que deberá articular sus acciones y recursos con las entidades del Estado, a fin de atender la problemática y garantizar algunos recursos profesionales y materiales para el inicio de acciones. El Ministerio de Salud, del Trabajo y Educación deben trabajar coordinadamente en la unificación de recursos para la rehabilitación y reinserción social y laboral: dotación de programas y recursos comunitarios y hospitalarios, a fin de evitar la cronificación y deterioro de muchos de éstas personas.

La principal responsabilidad debería recaer en los Gobiernos Locales, quienes deberían diagnosticar los alcances de éste fenómeno a través de un censo y en una etapa posterior indagar sobre algunas características personales básicas a través de la aplicación de una encuesta a una muestra de personas que vivan en esta situación. Información que resulta básica para la puesta en marcha de políticas públicas específicas para este sector de la población. La familia es un sector de población que debe asumir un mayor protagonismo en la atención de esta población dado de su carácter excluyente.

\section{Pertinencia y oportunidad}

No existe a nivel nacional un diagnóstico de las personas con capacidades físicas y mentales reducidas y en situación de calle, ni una base de datos que de cuenta de la oferta de demandas y servicios de atención y rehabilitación en el tema a nivel nacional. Las instituciones en beneficio de esta población están desarticuladas.

La reforma psiquiátrica reciente que activa la mayor expulsión de esta población a la calle por parte de la familia, ante la incapacidad de brindarles atención, cómo la ausencia de alternativas de atención que proponen. Se debe explotar la subsidiariedad del Estado para la atención de esta población y la red de servicios contratadas cómo población pobre y vulnerable sin capacidad de pago.

\section{Integralidad}

La reforma en la salud mental iniciada en Chile, si bien contempla la atención desde el Fondo Nacional de Salud a este tipo de pacientes, no contempla la atención y priorización de personas con limitación física y mental y en situación de calle, con mayores niveles de exclusión social y de cronicidad. Asimismo, las instituciones en las cuales se ha depositado la responsabilidad de su operatividad no cuentan con la dotación de recursos institucionales, locativos, materiales, financieros y humanos necesarios para la atención específica y expedita de estas personas. Debe ser 
responsabilidad de los Gobiernos locales el diagnóstico y la coordinación interinstitucional para garantizar el cumplimiento de la Política del Ministerio de Salud. El Ministerio de Educación y del trabajo deben generar condiciones mínimas que aseguren el acceso igualitario de esta población a las oportunidades formativas y laborales que el Estado pueda ofrecer.

\section{Coherencia}

La coherencia está dada por el reconocimiento de las personas en esta situación cómo sujetos sociales de derecho y no meros receptores de beneficios del Estado. Ello significa que es necesario el desarrollo de sus capacidades sociales necesarias para impulsar creativamente acciones de transformación social. Su especial situación los pone en una situación de mayor vulnerabilidad. En un grupo social en situación de extrema pobreza, por lo tanto, de mayor exclusión social. Es necesario generar una red social (Salud-Comunitario) eficiente que articule recursos y programas para la atención de esta población y que facilite la dinamización familiar en favor de la reinserción social de estas personas.

\section{Diseño de estrategias de la política}

Las opciones de esta propuesta están dirigidas hacia dos frentes pero tienen los mismos principios identificados en el problema. La intervención social con hombres entre 30 a 59 años, con limitaciones físicas y/o mentales en situación de abandono y exclusión social, busca que las personas atendidas mejoren su calidad de vida, mediante la satisfacción de las necesidades básicas, fortaleciendo los vínculos sociales creados a lo largo de su vida y la promoción del desarrollo de un proyecto personal de vida, con participación en las actividades institucionales y no institucionalizados. Se trata de garantizar que hombres y mujeres sujetos de esta política social continúen y fortalezcan su proyecto de vida con la responsabilidad y autonomía que la reducción de sus capacidades físicas y mentales le permita.

\section{Opción 1 :}

Creación de Centros de Atención Ambulatorios Diurnos: Esta atención consiste en proporcionar durante ocho horas diarias servicios de tipo psicosociales, de salud y de protección con base en la articulación de diferentes instituciones públicas y privadas, así como las redes sociales y familiares de los sujetos de la política.

El área de protección - Consiste en la satisfacción de necesidades básicas, tales como: Alimentación, higiene, vestuario, así como actividades ocupacionales, terapéuticas, recreativas y culturales que promuevan procesos de socialización en el marco de sus derechos. Se debe ofrecer un adecuado manejo de las diversas patologías que los usuarios presenten, igualmente controlar los factores de riesgos, relacionadas con el 
abandono y la indigencia, a fin de mantener y mejorar la salud individual y colectiva a través de condiciones dignas. Se estima que las áreas de atención en esta alternativa deben incluir acciones psicoterapéuticas, para evaluar y tratar la salud mental, el área socio-familiar, que a partir de un diagnóstico intenta revincular la familia de las personas en situación de abandono.

El área ocupacional - Elaborará un perfil ocupacional que identifique habilidades y destrezas de cada uno de los sujetos, a fin de habilitarlos en alguna tarea tendiente a su reinserción socio-familiar.

El área de salud - propenderá a garantizar la atención médica, terapéutica y psiquiátrica, mediante medicamentos y procesos de rehabilitación. Se contempla además, educación en derechos y deberes en el sistema y promoción de salud, dirigidas a la persona. Estos centros deberán ser responsabilidad de los gobiernos locales quienes deben proveer de los insumos para la puesta en marcha de los mismos, además deberán funcionar en coordinación directa con los consultorios locales y universidades de la región, quienes proveerán el recurso humano necesario para la atención de éstas personas.

\section{Opción 2:}

Reconstrucción de la red socio-familiar . Se trata de reconstruir y fortalecer la red social familiar de los sujetos de la política, para disminuir los factores de riesgo asociados con el maltrato y abandono de esta población, tratando de potenciar el desarrollo de sus habilidades y destrezas para favorecer su integración al medio social y familiar.

Estas acciones estarán acompañadas de suministro de apoyos asistenciales que incluyen atención médica y nutricional al sujeto, habilitación ocupacional que genere mayores niveles de autonomía a las personas y a su familia, asimismo mejorar las relaciones familiares y sociales. A nivel comunitario - se pretende potenciar redes de apoyo en los espacios educativos, ocupacionales, recreativos y culturales de la comunidad en que se inserta.

Esta opción deberá ser asumida por el Ministerio de salud, quien creará un Fondo Nacional e invitará a las Fundaciones, Ong's, universidades, entre otros a postular mediante una licitación abierta la presentación de proyectos para la revinculación de estas personas con sus núcleos familiares.

\section{Criterios de evaluación de la política}

Los criterios de evaluación de la política serán los siguientes: 
- Inserción social: Se refiere al acceso de estas personas al mercado de bienes y servicios (dimensión económica), acceso al empleo, a la protección social y la participación ciudadana (dimensión social), acceso a derechos humanos, políticos y civiles (etnia, identidad sexual, religión, género, características físicas y mentales).

- Integralidad: Referido a visión intersectorial superando de esta forma las limitaciones de las visiones solo sectoriales y que se articulen a partir de una perspectiva integral y holística, que se refleje en una coordinación y articulación eficiente y eficaz de la política pública, logrando así efectos sinérgicos.

- Equidad: Debe buscar compensar y corregir las exclusiones y/o desigualdades, en las diversas dimensiones en las que éstas se expresan: económica, de género, territorial y socio-cultural, entregando así un trato preferencial a las personas en esta situación.

- Pertinencia: Se requiere que la política que se ofrezca a estas personas contenga estrategias de intervención que respondan a las necesidades y particularidades de la realidad económica, social, cultural y geográfica donde ellos se desenvuelven y desarrollan.

- Participación: Se requiere involucrar a la mayor diversidad posible y número de sujetos de la política en las etapas de diagnóstico, evaluación de alternativas de solución y propuestas que se emprendan a favor de esta población.

- Dignidad: Se refiere al reconocimiento y ejercicio de los derechos económicos, sociales y políticos de los sujetos de la política

- Competencia local: Referido al reconocimiento de las competencias de las autoridades locales en la resolución de problemas presentes en su juridiscción.

- Trabajo en red: Hace referencia a la necesaria vinculación que debe existir entre el Estado y las instituciones públicas y privadas para la atención directa y prioritaria de esta problemática. Además la participación de la red familiar y comunitaria de esta población

- Apoyo al fortalecimiento de la familia: Se refiere a la permanente búsqueda de los recursos y potencialidades de las familias de origen de los sujetos de la política, tendientes a su habilitación para apoyar su proceso de reinsersión familiar y social.

- Sensibilización comunitaria: Referida a la vinculación que se hace con la comunidad directa a fin de lograr su participación activa en el proceso de reinserción social de estas personas, mediante acciones de promoción, prevención y fomento de actividades sociolaborales y de salud mental. 
Estrategia a seguir:

\begin{tabular}{|l|r|c|c|}
\hline CRITERIOS & \% & OPCION 1 & OPCION 2 \\
\hline Inclusión social & 20 & 20 & 20 \\
Integralidad & 5 & 5 & 3 \\
Equidad & 5 & 5 & 3 \\
Pertinencia & 5 & 5 & - \\
Participación & 10 & 10 & 10 \\
Dignidad & 15 & 15 & 15 \\
Competencia Local & 10 & 10 & 10 \\
Trabajo en Red & 15 & 15 & 10 \\
Apoyo al fortalecimiento familiar & 10 & 5 & 10 \\
Sensibilización Comunitaria & 5 & 5 & 5 \\
\hline TOTAL & $\mathbf{1 0 0}$ & $\mathbf{9 5}$ & $\mathbf{8 6}$ \\
\hline
\end{tabular}

El problema de las personas adultas con limitaciones físicas y mentales en situación de abandono y ausencia de redes sociales y familiares, es un tema invisibilizado ante la sociedad, las autoridades, la opinión pública y las propias familias, por lo cual puede ser abordado por ambas opciones lo importante es rentabilizar el tipo de inversión que se quiere realizar dependiendo de la factibilidad económica de la alternativa y por supuesto, del organismo y/o autoridades que le ejecuten.

\section{Diseño de implementación}

La implementación de la política se refiere a las del tipo de segunda generación, correspondiente al tipo adaptativa: "Top Down" "Down Top" (Van Meter \& Colls., 1993), ésta focaliza en los aspectos operacionales que determinan la provisión misma de los beneficios a los derechohabientes. Es un enfoque que adapta el curso de acción a los intereses y expectativas de beneficiarios y ejecutores. Es un enfoque que da espacio a iniciativas locales, está más centrado en el logro que en las formalidades del cumplimiento de instructivos y reglas. Es por ello que los actores participantes de la política, dependiendo de la acción tomada, algunos aparecen como facilitando u obstaculizando la ejecución de la misma.

En primer lugar se tendría el caso de los actores que actuarían de manera positiva como son: Los sujetos de la política (Hombres y mujeres entre 30 y 59 años con limitaciones físicas y mentales, abandonados y en situación de calle), las instituciones 
estatales, la sociedad civil, el tercer sector y las universidades locales. Se considera que uno de los actores que podría eventualmente actuar de manera negativa frente a la política sería la familia de estos sujetos, ante la amenaza de hacerse responsable de manera individual de la situación.

En tanto, la sociedad civil (redes sociales y ONG's), el Estado (ministerios e instituciones) y los gobiernos locales se constituyen en actores neutrales debido a que aún no visibilizan el problema pero ante la evidencia de esta realidad ellos podrían incorporarse activamente al diseño e implementación de la política, pues es un tema de su competencia. Igualmente, en el proceso de implementación hay que construir escenarios posibles de darse frente a los distintos actores que participarán teniendo en cuenta las ganancias y las pérdidas de cada actor frente a la opción tomada, para formular estrategias que contribuyan fortalecer las primeras y a contrarrestar o neutralizar las segundas. De este modo, es posible efectuar en detalle las mismas, estableciendo estrategias para atenuar su efecto:

Sujetos de la política - Las ganancias que es posible identificar en el caso de estos actores son las siguientes: Reconocimiento como sujeto de derechos (Maia y Rodríguez, $2006)^{4}$, Atención desde los servicios locales, Vinculación con familia, Reinserción sociolaboral, Autonomía. En tanto que las pérdidas serian las siguientes: Dependencia de la caridad pública, Consumo de drogas, Uso de espacios públicos. Para contrarrestar estos efectos se plantean estas estrategias: ofrecer servicios de alimentación, salud, recreación, vestuario y aseo desde la identificación de los mismos; postularlos en forma prioritaria a los subsidios estatales; gestionar red social de salud emergente; coordinar con autoridades locales el uso de los espacios públicos por estas personas; $\mathrm{e}$ iniciar campañas para sensibilizar a la población respecto a formas efectivas de ayudar a estas personas, sin entrega de dinero a los mismos.

Instituciones estatales (consultorios) - En éstas las ganancias serían: Hacer la atención directa, focalizada e integral, Priorizar recursos hacia uno de los sectores más excluidos de la sociedad, Adquirir experiencia en la atención en salud mental, Imagen institucional y social, Responsabilidad social de las instituciones, Capacidad institucional y capacitación de equipos multidisciplinarios. Las pérdidas se relacionan con: Dispersión de recursos, Visión excluyente y diferenciadora de esta población (perjuicios, mitos, etc.), Trabajo monótono y rutinario en la atención. Ahora, las estrategias que permitirían avanzar en las pérdidas que eventualmente se ocasionarían son: organizar la atención a esta población de forma integral, focalizada y priorizada; iniciar campaña de sensibilización al interior de los equipos de salud con el objeto de visibilizar los problemas que afrontan los sujetos de la política; realizar campañas de difusión de la existencia de la política y sus programas; y capacitar a los equipos profesionales vinculados en el tema de la salud mental y discapacidad.

${ }^{4}$ Estas autoras en su trabajo proponen empezar por dejar de llamarlos 'locos' y considerarlos ahora como sujetos portadores de trastorno mental', pretendiendo con ello, su reconocimiento como ciudadanos, sujetos de derechos. 
Ong's - Se destacan como ganancias para este sector las siguientes: Posibilidad de ejecutar políticas públicas sociales innovadores, Imagen institucional y social, Presencia en el ámbito local, Posibilidad de ejecutar proyectos pilotos en éste área y experiencia. Apropiación de recursos del Estado para desarrollar esta política, Empoderamiento en términos de la posibilidad de relación directa con el Estado, Crecimiento organizacional. Estas organizaciones no tendrían que verse afectados con la implementación de la política, muy por el contrario ésta sería una oportunidad para participar activamente en la propuesta, implementación y evaluación de la política pública. Las estrategias en este caso serían: organizar la atención a esta población de forma integral, focalizada y priorizada; sensibilizar a ONG's sobre el sentido de la política; vincular las ONG's con la red institucional local y nacional del Estado; diseño de convocatoria y licitación pública para la presentación de proyectos en esta área; y crear condiciones para le interlocución y negociación directa de éstas con el Estado (Programas, recursos, bases de licitaciones, etc.).

Los gobiernos locales - Estos serian los principales actores en la implementación de la política, por tanto estas son sus ganancias: diseñar políticas públicas focalizadas integrales; priorizar recursos hacia uno de los sectores más excluidos de la sociedad; imagen institucional y social; efectividad en la responsabilidad social de las provincias o comuna; capacidad de trabajar en red con instancias del nivel local; priorizar desde el diseño de la política a uno de los sectores más excluidos de la sociedad; avanzar en el tema de la seguridad ciudadana, ciudadanía y protección social a nivel local; credibilidad pública. Las pérdidas se vinculan con: visión excluyente y diferenciadora de esta población (prejuicios, mitos, etc.); posibilidad de asignar mayores recursos para otros programas sociales de la comuna y /o provincia; criticas de ciertos sectores políticos, sociales, institucionales y gremiales.

Las estrategias para neutralizar tales pérdidas se orientan a: iniciar lobby público que comprometa a las diversas autoridades locales; realizar campañas de difusión de la existencia de la política y sus prògramas (radio, televisión, prensa escrita); presentar estudios que avalen la rentabilidad social de la política; hacer "marketing" social de la política.

Sociedad civil - Las ganancias de éstas son: reconocimiento de la dignidad y derechos de todos los seres humanos; seguridad social y ciudadanía; reconocimiento y trabajo hacia poblaciones más vulnerables socialmente; oportunidad de hacer labor social frente a esta población; oportunidad vinculación laboral en esta área. Las pérdidas se relacionan con los siguientes factores: desinterés y exclusión hacia este grupo poblacional, 'estabilidad' social adquirida en su funcionamiento.

Las estrategias están dirigidas a: sensibilización para el reconocimiento de las diferencias y el derecho a la igualdad; educación en deberes y derechos en el sistema y promoción de la salud; vinculación como red de apoyo social; generación de un 
grupo de voluntarios para la atención social de esta población; y generación de ofertas laborales en esta área.

Universidades locales - Las ganancias para éstas son: nuevo espacio para la investigación y la gestión social, Mayor imagen corporativa y socia; generación de convenios de cooperación interinstitucional; contribuir a equidad y justicia social, Construcción de red institucional de apoyo; rol activo en la generación de políticas públicas; espacio para prácticas académicas; mayor vinculación entre la universidad y el contexto social; y "marketing" social de la universidad en el espacio público de generación de políticas. Las ganancias son muchas para este actor: estabilidad en el funcionamiento de su sistema académico y anonimato en la sociedad.

Las estrategias para lograr su mejor participación son las siguientes: cabildeo institucional para la oferta de políticas y programas sociales; invitación a participar en diseño de bases de convocatoria y licitación de programas y proyectos; ofertar espacios de prácticas profesionales; ofertar espacios para el desarrollo de líneas de investigación en esta área; y ofrecer espacio a la academia como evaluador del diseño, implementación y evaluación de la política pública

Actores neutrales - Es necesario integrarlos de manera decidida en la implementación de la política a través de estrategias que logren su cooperación activa, para este caso las que se plantean las siguientes:

Sociedad civil: Visibilizar el problema social que afecta a estas personas, vinculándola con el tema de la seguridad ciudadana y la recuperación de espacios públicos locales, a través del levantamiento y socialización de información acerca de esta realidad.

Ong's: Recopilación de la experiencia de trabajo de estas entidades con este tipo de población tendiente al diseño de propuestas de intervención con participación activa en la convocatoria de programas.

Ministerios públicos: Comprometer las voluntades políticas participantes de la agenda en la implementación de soluciones integrales que contemplen acciones de atención, promoción y prevención del problema a través de los mecanismos que el Estado ha establecido para este fin, a través del lobby diseñado oportunamente.

Instituciones estatales: Propiciar la alianza con instituciones privadas y fundaciones que trabajen en el tema, disponiendo recursos para la generación de propuestas de atención a la población.

Gobiernos locales: Generar mesas de trabajo en torno al tema especifico de la política con participación de representantes de organizaciones e instituciones para comprometer las autoridades locales en la identificación de prioridades, agenda de trabajo, recursos 
y mecanismos de financiación locales.

En toda política pública independiente del sector o autoridad que la convoque es necesario considerar un análisis detallado de los posibles contextos en los cuales se puede desenvolver un actor determinado, para de esta forma pensar en articulaciones ó alianzas que permitan salir adelante en la implementación de la misma. Para el caso de esta política a continuación se presentan detalladamente las organizaciones representativas con las cuales es necesario vincularse para la propuesta. 


\begin{tabular}{|c|c|c|}
\hline ORGANIZACIONES & ROL & FORMAS DE ARTICULACION \\
\hline FAMILIA & $\begin{array}{l}\text {-Representantes directos de los } \\
\text { intereses de esta población. } \\
\text {-Facilitador del proceso de reinserción } \\
\text { social y laboral de los sujetos de la } \\
\text { política. } \\
\text {-Protector del riesgo psicosocial de } \\
\text { estas personas. } \\
\text {-Demandante externo de la eficiencia } \\
\text { de la red institucional y social de apoyo. } \\
\text {-Organizador de movimiento social que } \\
\text { demanda la atención a esta población. }\end{array}$ & $\begin{array}{l}\text {-Mesas de trabajo. } \\
\text { - Fomento de organización social } \\
\text { que represente intereses de esta } \\
\text { población. } \\
\text { - Uso de subsidios estatales para } \\
\text { estas personas. } \\
\text {-Participante activo de la política } \\
\text { (demandante, ejecución y evaluación). }\end{array}$ \\
\hline SOCIEDAD CIVIL & $\begin{array}{l}\text {-Garante del cumplimiento de la } \\
\text { política social. } \\
\text {-Participe de la red de apoyo social. } \\
\text {-Participante activo del voluntariado } \\
\text { vinculado a la política. } \\
\text {-Facilitador del proceso de reinserción } \\
\text { social y laboral de los sujetos de la } \\
\text { política }\end{array}$ & $\begin{array}{l}\text {-Mesas de trabajo. } \\
\text {-Fomento de organización social } \\
\text { que apoye la reinserción } \\
\text { sociolaboral de esta población. } \\
\text {-Uso de subsidios estatales para } \\
\text { estas personas. } \\
\text {-Participante activo de la política } \\
\text { (demandante, ejecución y evaluación). }\end{array}$ \\
\hline ONG's & $\begin{array}{l}\text {-Diseñadores y ejecutores de la política. } \\
\text {-Participantes de la red institucional } \\
\text { de apoyo. } \\
\text {-Interlocutores directos con el Estado } \\
\text { y la familia. } \\
\text {-Evaluadores y asesores respecto a la } \\
\text { ejecución de la política. }\end{array}$ & $\begin{array}{l}\text {-Mesas de trabajo. } \\
\text {-Uso de subsidios estatales para } \\
\text { estas personas. } \\
\text {-Participante activo de la política } \\
\text { (ejecución y evaluación). }\end{array}$ \\
\hline $\begin{array}{l}\text { MINISTERIOS } \\
\text { PUBLICOS }\end{array}$ & $\begin{array}{l}\text {-Responsables directos de la } \\
\text { implementación de la politica. } \\
\text {-Gestores de los recursos necesarios } \\
\text { para la ejecución de la política. } \\
\text {-Gestores de la red institucional y } \\
\text { socialpara el desarrollo de la política. }\end{array}$ & $\begin{array}{l}\text {-Mesas de trabajo. } \\
\text { - Participante activo de la politica } \\
\text { (diseño, ejecución y evaluación). } \\
\text {-Convocantes de red } \\
\text { interinstitucional. }\end{array}$ \\
\hline $\begin{array}{l}\text { INSTITUCIONES } \\
\text { ESTATALES }\end{array}$ & $\begin{array}{l}\text {-Implementadores de la política. } \\
\text {-Participantes de la red institucional } \\
\text { de la politica. } \\
\text {-Oferentes de recursos para el } \\
\text { desarrollo de la política (subsidios). } \\
\text {-Convocadores y seleccionadores } \\
\text { de licitaciones para programas. } \\
\text {-Evaluación, control y supervisión } \\
\text { financiera y técnica de los programas. }\end{array}$ & $\begin{array}{l}\text {-Mesas de trabajo. } \\
\text {-Participante activo de la política } \\
\text { (ejecución y evaluación). } \\
\text {-Gestionar recursos ante el Estado, } \\
\text { la sociedad civil y organismos } \\
\text { internacionales. } \\
\text { - Agentes convocantes de ofertas de } \\
\text { intervención por parte de otras } \\
\text { instituciones interesadas en la } \\
\text { problemática. } \\
\text {-Recepción de información referente } \\
\text { a la forma de ejecución de la política } \\
\text { por parte de los demandantes. }\end{array}$ \\
\hline $\begin{array}{l}\text { GOBIERNOS } \\
\text { LOCALES }\end{array}$ & $\begin{array}{l}\text {-Generadores de recursos para ampliar } \\
\text { la caracterización de la problemática e } \\
\text { implementación de programas en cada } \\
\text { localidad. } \\
\text {-Participante activo de la red institucional } \\
\text { de la politica. } \\
\text { - Control y uso del espacio público como } \\
\text { garante de la seguridad ciudadana. }\end{array}$ & $\begin{array}{l}\text {-Mesas de trabajo. } \\
\text {-Oferte de beneficios sociales para } \\
\text { estas personas. } \\
\text {-Participante activo de la política } \\
\text { (ejecución y evaluación). } \\
\text {-Evaluadores externos de la } \\
\text { ejecución de la política. }\end{array}$ \\
\hline
\end{tabular}


Pensando que esta política pretende beneficiar a un sector poblacional cuyos actores carecen de formas de expresión y articulación social, es necesario que la sociedad civil a través e sus organizaciones revele esta demanda ante el Estado. Para ello previamente se requiere la planificación de una serie de actividades antes que el tema pase a la agenda pública, tales como las siguientes:

1. Lograr la visibilización de la situación de estas personas como un problema público y no como situaciones individuales y diferenciadas, por medio del levantamiento de información de tipo cuantitativa y cualitativa relacionada con la magnitud del problema en el orden nacional y las características del mismo. Se puede lograr con: levantamiento de un censo de la población afectada por el problema en el país; elaboración de un diagnóstico que de cuenta de la situación social, física y psicológica de estas personas; vincular a los gobiernos locales, a fin de que destinen recursos humanos y materiales para la realización de esta fase; y socializar los resultados del censo y del diagnóstico con autoridades competentes y en la comunidad en general.

2. Recoger la experiencia de las instituciones privadas, ONG's y voluntariados que han atendido de una u otra manera a las personas con capacidades físicas y mentales reducidas y en situación de abandono, a fin de reconocer la efectividad o no de su intervención. Se debe: identificar las instituciones que han respondido de manera emergente ante esta problemática en cada localidad; determinar las estrategias de intervención social y recursos utilizados para atender a esta población; establecer impacto de la atención brindada a esta población por parte de las instituciones identificadas; identificar aciertos y desaciertos en la atención; y recoger propuestas de intervención a partir de sus experiencias.

3. Propiciar la alianza de instituciones privadas y fundaciones que trabajen en el tema ya sea de capacidades físicas y mentales reducidas, en el de abandono y en el de extrema pobreza, ya sea en la atención directa ó que tengan algún tipo de responsabilidad social (salud, previsión, trabajo, educación) frente a estas personas, tendiente a estructurar una propuesta que demande desde el Estado acciones inmediatas de reinserción y habilitación social. Se sugiere: convocar a las diversas instituciones públicas, privadas y ong's, que trabajen en la temática a conformar un grupo de presión que interlocutor con el Estado, en relación a las demandas de esta población y la necesidad de respuestas inmediatas; evidenciar ante la opinión pública las carencias y necesidades de esta población; y colocar en el debate público este problema con ayuda de los medios de comunicación.

4. Crear estrategias de articulación entre estas instituciones con esferas de representación política tales como partidos políticos, sectores influyentes, empresarios, y opinión pública (medios de comunicación) en general, que 
permita presentar la dimensión social, humana, económica del problema y de esta manera generar mayor conciencia social del problema en el país. Algunas recomendaciones son: generar mesas de trabajos en torno al tema específico y sus diversas dimensiones, con la participación de representantes de organizaciones, instituciones, entre otras; diseñar propuestas viables de intervención que involucren la participación de los actores convocados en estas mesas; utilizar el espacio de las mesas de trabajo cómo mecanismo para identificar y comprometer asignación de recursos humanos, materiales, financieros para financiar las propuestas.

5. Diseñar una agenda de trabajo que vincule todos los sectores y actores involucrados en la atención del problema a fin de señalar escenarios posibles y estrategias de intervención social a corto, mediano y largo plazo que sean evaluadas de manera factible por una comisión de técnicos que represente a todos los actores participantes. Se sugiere: elaborar con los actores convocados la agenda de trabajo, que incorpore metas, presupuesto y cronograma de actividades; conformar una comisión de especialistas que representen a todos los sectores involucrados para el análisis de factibilidad del plan diseñado; y ajustar la agenda a la evaluación realizada por los especialistas de Política Pública.

6. Propiciar una organización representativa de todos los sectores y actores involucrados en la solución del problema ${ }^{5}$ con la finalidad de monitorear los avances e impacto de la política nacional diseñada para este sector poblacional. Para su logro se puede: establecer la ubicación de las familias de estas personas a través del Registro Civil, a fin de informarlas sobre la situación de sus parientes y la necesidad de conformar un tipo organización para movilizar necesidades e intereses; evaluar posibilidades reales de que las familias puedan asumir el cuidado y protección de sus parientes; definir apoyos requeridos por las familias y evaluar factibilidad de concretizarlos en acciones y recursos; crear una organización que agrupe a los familiares de esta población y las instituciones interesadas en demandar respuestas frente al problema; y dinamizar el funcionamiento de esta organización social tendiente a su interlocución efectiva ante las autoridades competentes.

\footnotetext{
${ }^{5}$ En este sentido se retoma el planteamiento de estas organizaciones como movimientos sociales según lo señala Alain Tourraine como " actores colectivos cuya orientación principal es en la defensa del sujeto, la lucha por los derechos y la dignidad humana....Un movimiento social no es una corriente de opinión, puesto que pone en tela de juicio una relación con el poder que se sitúa muy concretamente en las instituciones y en las organizaciones, teniendo la mira de orientaciones culturales, a través de las relaciones entre poder y desigualdad" (1997, citado por Eroles, 2006: 3)
} 
7. Comprometer las voluntades políticas participantes de la agenda en la implementación de soluciones integrales que contemplen acciones de atención, promoción y prevención del problema a través de los mecanismos que el Estado ha diseñado para tal fin. Se recomienda: elaborar un cronograma de encuentros con la autoridad pública donde se establezca la magnitud de la situación, alternativas de solución, compromisos de los diferentes actores, análisis de factibilidad de las soluciones; y monitorear el cumplimento de las estrategias destinadas a la incorporación del problema en la agenda pública.

\section{Evaluación}

Se deberá trabajar con un tipo de evaluación tanto, cualitativa y cuantitativa. La primera, se realiza a partir de la perspectiva de los sujetos beneficiarios, por lo tanto es subjetivista y emergente, haciéndose desde 'dentro', pues se orienta al reconocimiento de la persona como ciudadano sujeto de derechos y por lo tanto, se hace necesario la consulta individual a éstos respecto a la implementación de la política. Se hará con base en un modelo de tipo sensitivo, el cual tiene tres momentos:

1. Elabora plan de observaciones y transacciones con los usuarios de la política y su familia.

2. Evalúa la política con varios actores: ONG's, gobiernos locales, fundaciones, etc. Averigua que es valioso para el usuario y su familia, y reúne juicios de otras entidades y actores participantes eventuales de la política.

3. Se realiza una triangulación de a información entre los encargados de la política y los usuarios y de allí se realiza un informe final.

Las técnicas de recolección de información cualitativas que se utilizaran serán: Observación directa, grupos focales y entrevista en profundidad a una muestra representativa de la población atendida. La cual deberá contar con criterios como los siguientes: sexo, edad (30 a 59 años), limitaciones físicas, total abandono, en situación de calle y participante como beneficiario de la política.

En el caso de las personas con capacidades mentales reducidas, se realizarán grupos focales, los cuales se desarrollaran mediante una guía sobre la forma como evalúan la política que será discutida además con algunos miembros de su grupo de pares, con los que establece relaciones antes y durante la implementación de la política, y que puedan dar cuenta de un mejor estado de salud en este sentido. Esta técnica dará cuenta de su estado de salud, articulación con redes sociales y familiares y actitud. personal frente a la política. 
Además, el diseño de tipo cuantitativo, se realizará mediante una encuesta aplicada a la población beneficiaria y un registro que de cuenta de los siguientes tópicos:

-Número de personas beneficiarias.

-Número de familias que participan en el programa.

-Número de personas reinsertadas con su familia.

-Mejoramiento de salud física (tratamiento, síntomas) de cada beneficiario.

-Mejoramiento de salud mental (tratamiento, síntomas) de cada beneficiario.

-Número de centros de día que existen.

-Tipo de servicios con que cuentan.

-Número de redes sociales que se vinculan.

-Número de beneficiarios reinsertados laboralmente.

Los resultados que arroje la evaluación de la política, de acuerdo a los criterios antes señalados, deberán ser socializados a todos los actores participantes a fin de retroalimentar el proceso para fortalecer y/o modificar las acciones que sean necesarias por parte de cada uno. 


\section{Referencias}

Van Meter, Donalds S. \& Colls. (1993). El proceso de implementación de las políticas. Un marco Conceptual. En Luis Aguilar, (Ed). La implementación de las políticas. (pp. 97-147). México D.F.: Miguel Ángel Porrúa Grupo Editorial.

Camps, Victoria. (2001). Elementos Históricos del concepto de lo público. En: Memorias del V Encuentro Iberoamericano del Tercer Sector. Lo público, una pregunta desde la sociedad civil. (pp. 65-73). Bogotá: Quebecor Impreandes.

Eroles, Carlos. (2006). Los procesos de extinción-exclusión social, afectan en América Latina a los grupos más vulnerables, considerados población sobrante. En: Memorias $33^{\circ}$ Congreso Mundial de Escuelas de Trabajo Social. Santiago de Chile: Comité Organizador.

García Canclini, Néstor. (2001). La globalización imaginada (Segunda Reimpresión). Argentina: Editorial Paidós.

Iamamoto, Marilda. (2002). Fenómeno social de la exclusión. En: Sonia Severini (Coord.). Trabajo Social y Mundialización. Etiquetar desechables o promover inclusión. (pp. 28-40). Buenos Aires: Espacio Editorial.

Luhmann, Niklas, (1991). Sistemas sociales. Lineamientos para una teoría general. México D.F.: Universidad Iberoamericana, Alianza Editorial.

Maia, Ana Isi y Rodríguez, Roberta. (2006). De louco a portador de trastorno mental: A conquista de dereitos. Memorias $33^{\circ}$ Congreso Mundial de Escuelas de Trabajo Social. Santiago de Chile: Comité Organizador.

Ministerio de Palnificación y Cooperación (MIDEPLAN) (2003). Censo de Personas en Situación de la Calle. Santiago de Chile: Autor.

Pérez, Liliana, Soto, Rosa y Muñoz, Cinthia. (2004). La intervención social. Lo improbable del Trabajo Social hoy. Revista Palobra, Palabra que obra, 5, 12-18.

Pérez, Liliana (2005). Potenciamiento de la autonomía en los miembros de la familia: una proposición para asumir el riesgo en la sociedad contemporánea. Revista Palobra, Palabra que obra, 6, 123-136.

Pérez, Liliana y Cogollo, Kenia. (2006). Un ejercicio de develamiento de las representaciones de los jóvenes en las políticas sociales y en la modernidad. Revista Colombiana de Trabajo Social, 20, 195-211. 
Pérez, Liliana y Bolaño, Nancy. (2008). Memorias del territorio. Hacia políticas e intervenciones sociales complejas frente al desplazamiento forzado. Revista Prospectiva, 13, 175-223.

Tourraine, Alain. (1997). ¿Podemos vivir juntos? La discusión pendiente: El destino del hombre en la aldea global. Buenos Aires: FCE de Argentina. 